\title{
El concepto de ilusión en Schopenhauer y Nietzsche
}

\author{
MARCO PARMEGGIANI
}

El problema de la ilusión es una temática compartida por Schopenhauer y Nietzsche; pero no como aspecto secundario, sino como preocupación central. Constituye uno de los influjos claros del primero sobre el segundo, aunque Nietzsche desde luego replanteó el conjunto de la temática mediante otras ideas y bajo otros enfoques (el perspectivismo, la teoría evolucionista del conocimiento, el conocimiento como interpretación, etc). El concepto de ilusión (Schein, Illusion), el núcleo de esta temática, se halla en el trasfondo de casi la totalidad de sus respectivas filosofías, hasta tal punto que también es importantísimo para las reflexiones éticas, estéticas, culturales, etc. De ahí que para cualquier investigación que persiga una visión de conjunto de cada uno de sus pensamientos, o una penetración audaz en alguna cuestión concreta, es muy interesante disponer ante todo de una esclarecimiento del concepto de ilusión. Mi propósito no es desarrollar una comparación propiamente dicha de los significados de 'ilusión' en ambos autores, sino que me limitaré a mostrar el punto hacia el que ambos convergen. Defenderé la hipótesis de que los conceptos schopenhaueriano y nietzscheano de ilusión poseen un mismo esquema básico formal, aunque el desarrollo de este esquema difiera luego ampliamente. Además, intentaré demostrar que este esquema básico deriva directamente de Kant, es decir, no es más que el concepto kantiano de ilusión trascendental, si bien aplicado a nuevos ámbitos. Esbozado sintéticamente, el principal argumento que usaré para defender mi hipótesis será: sólo si interpretamos ambos conceptos de ilusión según el concepto kantiano, podemos resolver una serie de importantes paradojas o inconsistencias que se presentan en las filosofías de Schopenhauer y Nietzsche.

El concepto de ilusión está en el trasfondo de la toda la dialéctica trascendental en la Crítica de la razón pura. El objetivo de ella es justamente llevar a cabo una crítica de las ilusiones que asedian a la razón humana y que la conducen una y otra vez al error. Kant elabora ahí un concepto de ilusión (Schein), como distinto a apariencia (Erscheinung), muy innovador y lleno de consecuencias fructíferas. Él distingue la ilusión trascendental de la ilusión lógica. Ésta consiste en la simple imitación de la forma de la razón, como ocurre en la 
ilusión provocada por los silogismos sofísticos. Y su única causa es la carencia de atención puesta en las leyes lógicas. Por tanto, en el momento en que ponemos atención y relacionamos el caso en cuestión con esas leyes, la ilusión lógica se disuelve completamente. En cambio, la ilusión trascendental no desaparece aún cuando ha sido descubierta, aún después de que la crítica trascendental haya descubierto patentemente su nulidad. Por consiguiente, la ilusión trascendental es un tipo de ilusión que puede ser reconocida como tal, pero que propiamente no puede ser evitada en modo alguno, como ocurre en las ilusiones ópticas. Kant utiliza como término de comparación la ilusión óptica, y pone varios ejemplos, entre ellos el del astrónomo que, a pesar de toda su ciencia, no puede evitar que la Luna le parezca más grande en su salida. Kant llega incluso a otorgar el calificativo de 'natural' a esta ilusión y a la dialéctica que generan. Las ideas de Dios, yo y mundo no son unas ilusiones generadas por la incompetencia de los filósofos a lo largo de la historia, como a menudo se le ha reprochado a la teología, a la psicología racional y a la cosmología. Pero tampoco son, como se ha criticado otras veces, y se sigue haciendo, ilusiones generadas por la mala fe de algunos pensadores que urdieron artificios para engañar al ser humano. Por el contrario, esas ideas son ilusiones inherentes a la razón humana, que aún después de ser descubiertas siguen produciendo engaño sobre el entendimiento humano ${ }^{1}$.

¿Qué cometido le cabe entonces a la crítica trascendental? En primer lugar, intentará desvelar la ilusión de los juicios trascendentales, y en segundo lugar, intentará impedir que esta ilusión sigan engañando al pensamiento. Pero la crítica trascendental no podrá conseguir que esas ilusiones se disuelvan y dejen de ser ilusiones, en todo caso tendrá que estar siempre alerta: las ilusiones trascendentales no cesarán de engañar al entendimiento humano y de conducirlo a nuevos errores, de modo que los errores producidos por estas ilusiones no pueden ser eliminados de una vez, sino que una y otra vez hay que volver a removerlos ${ }^{2}$.

Kant llama al contenido de estas ilusiones trascendentales 'ideas'. Dentro de la variedad grandísima de significados de este término, él le da un significado peculiar: «Entiendo por 'idea' un concepto necesario de la razón del que no puede darse en los sentidos un objeto correspondiente» ${ }^{3}$. En la medida en que las ideas trascendentales son conceptos puros que sobrepasan los límites de la experiencia, no puede haber nunca un objeto adecuado a ellas. De este modo, el efecto engañador propio de las ilusiones trascendentales es hacer

${ }^{1}$ I. Kant, Crítica de la razón pura, ed. P. Ribas, Alfaguara, Madrid, 1988, «Dialéctica trascendental», Introducción I: «La ilusión trascendental», pp. 297-300.

${ }^{2}$ Ibid.

${ }^{3}$ I. Kant, Crítica de la razón pura, «Dialéctica trascendental», lib. I, sección II: «Las ideas trascendentales», p. 318. 
creer que hay un objeto (Dios, yo, mundo) donde en realidad no lo hay, donde en realidad sólo está la multiplicidad fenoménica.

Podemos extraer así de Kant un concepto formal básico, esquemático, de ilusión, que utilizaremos a lo largo de este trabajo. Sintetizándolo al máximo, caracterizaré la ilusión como un contenido cognitivo que:

(a) Es falso.

(b) Siendo falso, parece, se muestra sin embargo como verdadero.

(c) Por diversas causas, esta fuerza persuasoria suya es tan intensa que, incluso después de haber sido reconocida su falsedad, sigue pareciendo verdadero y, en caso de que el escrutinio crítico relaje la guardia, sigue llevando a engaño.

(d) Su falsedad no puede ser entendida de manera usual: lo que le ocurre no es que ese contenido cognitivo no se corresponde adecuadamente con el objeto, sino que no hay objeto alguno al que corresponderse, puesto que la ilusión crea un objeto donde en realidad no lo hay $\mathrm{y}$, lo que es más importante, ni siquiera lo puede haber (no es una cuestión empírica, sometida a lo contingente, ya que no puede haber objeto de lo incondicionado, según Kant, en sus tres modalidades, Dios, yo, mundo).

\section{ILUSIÓN, REPRESENTACIÓN Y VOLUNTAD EN SCHOPENHAUER}

Schopenhauer utiliza poco el término mismo de 'ilusión'. En su significado más radical siempre hace referencia al concepto hindú de 'maya': la potencia creativa de lo absoluto (Brahma) que produce el mundo como un mágico efecto ilusorio de sí mismo ${ }^{4}$. Pero aunque lo utilice explícitamente pocas veces, el concepto de ilusión subyace a toda la metafísica schopenhaueriana y sobre todo a esa duplicidad ontológica que da título a su obra capital: el mundo como voluntad y representación.

El texto principal donde Schopenhauer intenta explicar y demostrar el carácter aparente, de representación, del mundo, es el libro primero del primer volumen de su obra capital. De ahí partiré para, mediante un análisis de sus líneas fundamentales, intentar extraer unas cuantas ideas claras sobre la ilusoriedad del mundo. Con ellas podré demostrar cómo, a pesar de las apariencias, Schopenhauer utiliza al fin y al cabo el mismo concepto de ilusión que Kant. Veremos además que darse de cuenta de ello permite depurar su metafísica de numerosos malentendidos a los que se ha visto sometido su pensamiento, que lo convertían en una especie de cosmología de una energía primordial.

\footnotetext{
${ }^{4}$ A. Schopenhauer, Die Welt als Wille und Vorstellung [de ahora en adelante W], en Sämtliche Werke, ed. A. Hübscher, Brockhaus, Wiesbaden, 1972 , vol. I, $\$ 3$ p. 9, §5 p. 20, \$51 p. 299, §53 p. $322, \S 54$ p. $335, \S 60$ p. $389, \S 63$ p. 416.
} 
Ateniéndonos al comienzo de su obra capital, Schopenhauer parte de la idea siguiente: el mundo es mi representación ${ }^{5}$. Esta proposición tan sencilla es en realidad compleja de entender. ¿Qué significa? En principio que el mundo sólo existe como dato cognitivo de mis facultades mentales.

Por lo pronto hay que precisar qué significa 'mundo'. Cuando hablamos de mundo, por lo corriente hablamos del mundo tal cual se me hace presente, por la simple razón de que el término 'mundo' designa la totalidad de aquello que se nos aparece de modo inmediato. Pero se dirá, muchas veces pensamos en el mundo, no sólo como lo que se hace presente, sino también como cierta estructura profunda, que aunque no se muestre de manera inmediata, sí es el verdadero ser del mundo. ¿Se refiere Schopenhauer también a este segundo significado del termino? Esta pregunta sólo podremos responderla cuando hayamos avanzado más en su teoría del conocimiento. Por ahora, podemos tomar constancia de que, tal como dice en el primer párrafo, Schopenhauer se refiere a lo que se nos aparece (el sol, la luna, la tierra, las estrellas, etc). Ahora bien, la fuerza de la tesis schopenhaueriana es que no se refiere sólo a esos objetos en cuanto que aparecen, o sólo a su fenómeno, es decir, no sólo a la impresión que esos objetos producen en nuestros sentidos. En este caso la tesis sería tautológica: la impresión que causa el mundo en mis capacidades cognitivas sólo existe en la medida en que impresiona mis facultades cognitivas.

Para superar esta dificultad, hay que prestar atención a los matices formales de la exposición en este comienzo de su obra capital. Creo que Schopenhauer toma como punto de partida cierto 'realismo ingenuo' (no tematizado filosóficamente), que considera sobreentendido por la mentalidad general. Bajo un punto de vista estilístico, el comienzo del primer libro está redactado buscando un efecto retórico: se sobreentiende en el lector una mentalidad tácita, contra la que se dirige de manera contradictoria la primera frase y su explicación subsiguiente. Hay que dilucidarla brevemente para entender bien el significado de la tesis schopenhaueriana. Ese realismo ingenuo puede sintetizarse en dos ideas:

(i) Los objetos que se nos aparecen, en el pasado, presente y futuro, tienen cierta 'constitución en sî́ que causa la impresión que tengo en mis facultades cognitivas.

(ii) En principio, esta constitución en sí, igual que la impresión que produce, es sensible, es decir, a su vez puede ser llegar a impresionar mis facultades.

Por tanto, cuando hablamos del mundo como la totalidad de todo lo que aparece, es en definitiva la totalidad de la experiencia posible en sentido kantiano, pues también esa constitución en sí pertenece, por lo menos para esa

${ }^{5}$ Sobre estas primeras páginas de El mundo y su proposición inicial, cf. un fecundo estudio en A.I. Rábade Obradó, Conciencia y dolor. Schopenhauer y la crisis de la Modernidad, Trotta, Madrid, 1995, pp. 92-104. 
mentalidad común, a esa experiencia posible. Todo esto significa que el 'aparecerse' podría entenderse entonces tanto inmediata como mediatamente, es decir, aquél que se produce mediante la técnica y la ciencia ¿Y se refiere también Schopenhauer al modo de representarse la esencia de las cosas que tiene la metafísica, por ejemplo, la causa formal aristotélica?. En principio no. Schopenhauer sólo engloba bajo esa mentalidad general, como aclarará más adelante en el libro, a la representación científica, en la que siempre se trata de impresión a los sentidos, de apariencia sensible. Schopenhauer determina esta forma del fenómeno como representación intuitiva.

En conclusión, una buena forma de precisar la famosa tesis inicial de $E l$ mundo sería formulándola así: el mundo tal como se nos aparece a los sentidos, pero no sólo inmediata sino también mediatamente -a través de la técnica y la ciencia-, es mi representación.

¿Cuáles son las razones principales que aporta Schopenhauer para apoyar su idea? En diversos pasajes de sus obras, Schopenhauer se remite a los resultados de la Crítica de la razón pura, sobre todo a la estética trascendental, pero en este primer libro el argumento utilizado es distinto, y más bien procede de Berkeley ${ }^{6}$. Se podría formular de varias maneras: El mundo tal cual se hace presente a mis facultades cognitivas es objeto, pero sólo puede haber objeto en la medida en que es construido por un sujeto cognoscente. Dicho de otro modo, el mundo es la totalidad del aparecer, pero aparecer es siempre aparecer a algo, a un sujeto. Dicho en una formulación más sintética y abstracta: todo objeto sólo es tal, es decir, sólo existe como tal objeto en la medida en que existe para un sujeto.

A este argumento se le puede hacer una réplica fácil: estamos de acuerdo en que el objeto cognitivo sólo existe como tal siendo conocido por el sujeto, pero es una paso en falso lógico pasar de ahí, sin más argumentos y sólo con la impresión retórica, al plano ontológico; del hecho de que el sujeto constituye al objeto en cuanto conocido por él, no puedo deducir que lo constituya también en sí mismo. Sin embargo, el argumento berkeleyano lleva ya consigo una contrarréplica: pero ¿qué se quiere decir cuando se afirma 'que lo constituya en sí mismo'? Sólo caben dos posibilidades. Si lo volvemos a entender con la objetualidad, igual que al aparecerse, caemos en una sinsentido: 'en sí mismo' significaría independientemente de su ser conocido, pero ¿cómo puedo hablar yo de algo independientemente de que lo conozca? ¿Cómo puedo representarme algo independientemente de que me lo represente? Schopenhauer ataca repetidas veces con este argumento al realismo: en el objeto no puede

${ }^{6}$ «Mas Berkeley fue el primero en expresarla resueltamente, y adquirió así un mérito inmortal en la filosofía» W I §1, pp. 4-5. En los suplementos al mundo explica más extensamente la aportación del idealismo berkeleyano, incidiendo continuamente en el argumento que voy a desarrollar, W II cap. I, pp. 4-5. 
quedar ningún residuo que yo no pueda representarme, en la medida en que de alguna manera ya desde el principio me lo estoy representando, ya por el mero hecho de hablar de él es ya objeto para un sujeto. La otra posibilidad sería entender el 'en sí mismo' fuera de toda objetualidad, y entonces me estaría vedado hablar de objetos en sentido alguno. Por ahora dejemos aquí esta alternativa, que nos obligaría a avanzar en la teoría del conocimiento schopenhaueriana hacia un terreno donde todavía no disponemos de elementos.

Existe la posibilidad de plantearle al argumento berkeleyano otra objeción, que quizás tenga más fuerza. Aceptemos que todo objeto es siempre en relación a un sujeto, aunque sea en una situación futura o pasada. Pero insistir en este hecho, en que todo objeto puede ser objeto conocido por un sujeto, desvía la cuestión de lo fundamental: ¿qué determina preponderantemente a ese ser conocido del objeto: la realidad en sí, el sujeto, ambos? En realidad, Schopenhauer ataca contra un falso enemigo, parte de un mal planteamiento de la cuestión: no se trata de saber si el objeto puede darse completamente fuera de su posible ser conocido, sino de establecer, de manera convincente, quién determina de forma preponderante ese ser conocido: el objeto conocido. Si lo hace el sujeto, entonces tendremos que concluir la gratuidad de postular un ser en sí del objeto, a parte de su ser conocido. Si lo hace el objeto, entonces no habrá justificación para negar la realidad en sí del objeto: porque puede ocurrir perfectamente que el objeto sea siempre objeto conocido, pero donde lo que determina su ser conocido es la realidad en sí del objeto, y no el sujeto. Como ejemplo de este último caso pongámonos en el realismo científico más débil: no se niega que todo aquello de lo que hablo o puedo hablar sea teoría, idea, pero eso no quita que, por otras razones, tengamos que pensar que esas ideas o teorías dependen en lo fundamental (en última instancia se aceptan o no) de una realidad exterior a ellas, en función de las respuestas que la realidad extramental dé a los experimentos y observaciones?

Por ahora no voy a intentar responder a esta objeción, sino todo lo contrario, la dejaré abierta porque me parece iluminadora. La tomaré de referencia para seguir aclarando y comprobando la validez del argumento berkeleyano. Hemos concluido así provisionalmente que es muy importante distinguir dos cuestiones:

(a) En qué medida el objeto es siempre conocido por un sujeto.

(b) Qué instancia, en el acto cognitivo, juega una papel determinante: el sujeto y sus estructuras, o el objeto y sus estructuras.

${ }^{7}$ Este realismo científico, más débil con respecto al metafísico, lo hallamos explicado y defendido inmejorablemente en A. Diéquez Lucena, Realismo científico. Una introducción al debate actual en la filosofía de la ciencia, Universidad de Málaga, Málaga, 1998. 
Es evidente que entre ambas, la cuestión clave es la segunda. Esta distinción se corresponde con la distinción de dos tipos de tesis idealistas. ¿Qué significa que el objeto sólo es tal para un sujeto?:

(a) Lo que llamaré tesis débil del idealismo: que el objeto, si no es conocido ahora de hecho, es siempre algo que puede ser conocido, que será conocido en el futuro, aunque esa posibilidad no llegue nunca a realizarse por circunstancias accidentales. Por ejemplo: planetas que ahora no se conocen, tiene sentido que hablemos de ellos como existentes porque pueden ser conocidos.

(b) Lo que llamaré tesis fuerte del idealismo: que el objeto sólo existe en cuanto conocido por un sujeto.

La primera tesis resulta evidente porque es la más débil, mientras que la segunda es la tesis fuerte del idealismo, la que necesita más argumentos y más contundentes. La dificultad para la argumentación idealista es que propiamente nada tienen que ver una con otra, y no es legítimo el paso automático de la primera a la segunda. Del hecho evidente de que todo objeto es cognoscible (aun teniendo en cuenta la posibilidad de impedimentos que serían accidentales) no se puede deducir sin más que el objeto sólo existe como conocido. Una razón en contra, que surge en seguida, es que calificar a un objeto de cognoscible lleva como presupuesto el inverso de la tesis fuerte: que existe ya antes del acto cognitivo. Esto significa que no sólo la primera no apoya a la segunda sino que la excluye. Lo extraño es que en las primeras páginas de $E l m u n d o^{8}$, Schopenhauer utiliza esta argumentación, y pretende partir de un juicio analítico sobre la relación sujeto-objeto, de tal manera que la misma definición de objeto incluya la necesidad de quedar reducido al ser conocido por un sujeto. Evidentemente todo depende de cómo entendamos objeto, y si lo hacemos según conviene a la tesis idealista, estamos dando por supuesto la solución del problema: presuponiendo lo que se quiere demostrar.

Vemos así que el análisis del argumento de Berkeley no conduce a resultados satisfactorios, de manera que su aparente fuerza persuasiva se diluye o en tautologías o en pasos lógicos incorrectos. Pero ¿por qué esta vía de análisis termina aportando muy poco al esclarecimiento del concepto de ilusión en Schopenhauer? ¿Por qué ocurre esto si hemos partido de la idea básica con la que él arranca el desarrollo de su obra capital?

Permítaseme plantear la hipótesis de que esa idea inicial, «el mundo es mi representación», no constituye en realidad el punto cardinal del concepto schopenhaueriano de ilusión. Ha resultado patente que ahondando en ella y en

\footnotetext{
${ }^{8}$ Schopenhauer además reitera el argumento en el suplemento correspondiente.
} 
el argumento berkeleyano conexo, no llegamos a resultados fructíferos respecto al concepto de ilusión. Quizás el problema es que le damos demasiada importancia al hecho de que Schopenhauer empieza su obra capital con esa tesis y ese argumento.

Propondré como tesis central del sistema schopenhaueriano lo que podríamos llamar la 'tesis kantiana', cuya centralidad sería lugar para otro trabajo demostrar. La tesis kantiana afirma la diferencia entre lo ideal y lo real, que es la manera que tiene Schopenhauer de formular la distinción entre fenómeno y cosa en sí. La peculiaridad de esta fórmula es que en cierto modo representa una 'ontologización' de la distinción kantiana. Es decir, Schopenhauer le confiere sentido ontológico a un planteamiento que tenía en Kant un estricto sentido gnoseológico. La diferencia radical entre lo ideal y lo real quiere decir que por una parte lo real nada tiene que ver con lo ideal, hasta el punto de que son formas de ser radicalmente distintas. Y viceversa, por otra parte, lo ideal, por propia esencia, nada tiene que ver con lo real.

Podríamos hacer una paráfrasis de la formula Schopenhaueriana que quizás se preste a menos confusiones: entre lo conocido (ideal) y lo existente (real) no hay una diferencia relativa que dejaría subsistir ciertas dependencias entre ellos, sino una diferencia radical que es como una fractura, donde no permanece ningún tipo de contacto entre ambos. Lo conocido, o lo que se puede conocer, no tiene ningún punto de contacto, nada en común, con lo que existe realmente y no sólo en cuanto conocido. Esta distinción deriva claramente de la diferencia kantiana entre cómo se nos aparecen las cosas (fenómeno) y lo que las cosas son en sí mismas (cosa en sí), y de su tesis principal acerca de la relación que ambos aspectos guardan entre sí: las cosas tal y como se nos aparecen (lo ideal) no pueden ser nunca como son en sí mismas (lo real).

Si tomamos entonces como tesis central la tesis kantiana, paralelamente habrá que dejar de lado el argumento berkeleyano y recurrir al argumento que en realidad es el más utilizado por Schopenhauer, el kantiano9. Tal y como lo plantea nuestro filósofo, podríamos sintetizarlo en el «argumento del filtro». Schopenhauer repite numerosas veces en su obra, que entre el sujeto y la realidad se interpone el entendimiento con sus estructuras, de modo que no percibimos ni conocemos la realidad cómo es en sí, sino cómo es según esas estructuras del entendimiento: es decir, aunque el contenido, las sensaciones, sí proceden de la realidad en sí, toda su organización hasta el más mínimo detalle es la impronta de la estructura del entendimiento, de manera que lo que conocemos en realidad son sus estructuras. «Pues si nuestra intuición, es decir, toda la aprehensión empírica de las cosas que se nos presentan, está ya

${ }^{9}$ B. Magge también defiende, con otros argumentos, la diferencia del idealismo schopenhaueriano respecto al de Berkeley, cf. Schopenhauer, Cátedra, Madrid, 1991, pp. 8389. 
esencial y principalmente determinada por nuestra facultad de conocer, y está condicionada por sus formas y funciones, entonces sólo puede ocurrir que las cosas se presenten de manera enteramente distinta a la de su propia esencia, y se muestren como con una máscara, que sólo hace presumir lo que se esconde debajo, pero nunca permite conocerlo; por lo que se trasluce como un misterio impenetrable y la naturaleza de cualquier cosa no puede pasar nunca enteramente y sin reservas al conocimiento» ${ }^{10}$. En este argumento el entendimiento es entendido como un filtro: entre la realidad y el sujeto cognoscente, de modo que nosotros no conocemos cómo son las cosas en sí mismas, sino únicamente cómo son para nosotros (para nuestro entendimiento). La estructura del mundo, que es lo que intentan conocer todas las ciencias, no deriva del mundo, sino de aquello que percibe el mundo.

Pero ¿puede interpretarse realmente así la función del entendimiento en la gnoseología schopenhaueriana? ¿dentro del conjunto de su filosofía es sostenible esta concepción? Mostraré seguidamente las dificultades que presenta este argumento. El concepto de filtro contiene tres elementos:

(1) Hay un objeto que se muestra tal cual es.

(2) Nosotros en principio podemos captar bien lo que se nos muestra, es decir, las capacidades cognitivas del sujeto son básicamente perfectas.

(3) Pero en medio de objeto y sujeto se interpone algo que modifica la cognición que tiene el sujeto del objeto y hace que al final el sujeto se forme una imagen cognitiva equivocada del objeto.

En propiedad, el concepto de filtro procede de ámbitos muy distintos, de la química y de la óptica. En la química, consiste en un dispositivo que funciona dejando pasar sólo algunos componentes de una disolución (líquida o gaseosa) y no deja pasar otros, de manera que el compuesto resultante difiere del de origen. Las características del filtro químico son así tres: (1) es un dispositivo que actúa de manera pasiva; (2) actúa sustrayendo y no adicionando; (3) y por tanto el medio de origen y el medio resultante son esencialmente homogéneos.

Algo muy parecido ocurre en el filtro óptico, que es el caso que tiene en mente Schopenhauer cuando arguye que no conocemos cómo son las cosas en sí mismas, porque entre ellas y nosotros se interpone el entendimiento -como un cristal coloreado nos da una visión alterada de los colores de un paisaje ${ }^{11}$. ¿Pero es realmente adecuado este símil para entender la función del entendimiento en la gnoseología schopenhaueriana, aunque él lo utilice? Resaltaré tres diferencias básicas entre el filtro y la función del entendimiento:

${ }^{10}$ W II cap. XVIII, p. 218.

${ }^{11}$ «Estas formas [espacio, tiempo y causalidad] legislan absolutamente sobre este mundo, de manera que toda experiencia tiene que conformarse siempre a ellas, del mismo que todo lo que veo a través de un cristal azul tiene que aparecer azul» A. Schopenhauer, Die beide Grundprobleme der Ethik, en Sämtliche Werke, vol. IV, p. 121. 
(1) El entendimiento no es pasivo, es activo: no se limita pasivamente a dejar que algo no pase y algo pase a través de él.

(2) El entendimiento añade: no sólo sustrae sino que añade algo nuevo.

(3) Por tanto, en principio hay muy poca homogeneidad entre el ámbito de origen y el resultante.

Estas tres características se adaptan perfectamente a la concepción que tiene Schopenhauer del entendimiento. Concretando, su función básica es organizar los datos de los sentidos, aplicándoles el principio de razón suficiente en sus cuatro versiones. Dejemos a un lado los argumentos aducidos con el fin de demostrar que el entendimiento 'pone' las formas del espacio, el tiempo y la causalidad, para organizar los datos de los sentidos que en sí mismos no poseen. Son básicamente los argumentos de la Crítica de la razón pura, que Schopenhauer se limita a tomar como punto de partida. Y sigamos la comparación con el filtro, ¿qué forma tiene aquello a lo que se aplica el entendimiento? Datos sensibles a los que no se les ha aplicado todavía lo más mínimo el principio de razón suficiente: puras sensaciones sin ningún tipo de espacio, tiempo, causalidad o devenir, ni organización ni unificación. Por consiguiente, el entendimiento no sólo sustrae, sino que añade elementos absolutamente novedosos respecto a los datos de la sensación: el espacio, el tiempo, la causalidad y a través de ellos toda la 'objetualidad'. Además, el ámbito de origen, al que se aplica el entendimiento, es por naturaleza completamente diferente del ámbito resultante. No hay ningún tipo de homogeneidad como ocurre en el caso de los filtros químicos y ópticos. De la comparación entre las funciones del filtro y del entendimiento hay que concluir que aducir el símil del entendimiento como filtro para argumentar la diferencia entre lo ideal y lo real resulta un tanto improcedente.

Permítaseme antes que nada extraer la conclusión interpretativa que íbamos buscando acerca de la ilusoriedad del mundo. Recordemos las tesis: «el mundo es mi representación» significa que todo objeto es siempre objeto para un sujeto. Vimos que no es correcto pasar directamente de la constatación de que todo objeto es siempre algo conocido por un sujeto, a la conclusión de que el sujeto construye cognitivamente al objeto. Esta segunda es la tesis fuerte del idealismo y en ese sentido hay que entender la tesis ambigua de que todo objeto es siempre objeto para un sujeto. Ahora hemos descubierto el único argumento posible a favor de esta tesis fuerte, que no es el argumento berkeleyano. En el acto cognitivo juega un papel ampliamente preponderante el sujeto frente al objeto, porque es el entendimiento quien organiza el material bruto dado por el objeto, mediante sus formas básicas del espacio, tiempo y causalidad. Como no podemos imaginarnos un dato cognitivo sin forma alguna, el aporte del objeto, las puras sensaciones, sufre una especie de reducción infinitesimal, algo parecido a lo que ocurre en el límite matemático. Por 
esta razón, y no por otra, todo objeto es siempre objeto para un sujeto, y el mundo tal como se nos aparece a los sentidos, pero no sólo inmediata sino también mediatamente, es mi representación.

He extraído así un esquema básico acerca de la ilusoriedad del mundo: lo aportado propiamente por el objeto son las puras sensaciones, mientras que el sujeto es quien añade la organización a través de una serie de formas básicas que le son intrínsecas, y así construye el mundo objetivo. Sin embargo me parece que aún esta conclusión presenta importantes dificultades teoréticas. Básicamente son dos:

(i) El objeto sólo es objeto en cuanto que el sujeto, mediante sus formas, así lo construye, entonces ¿cómo eso construido por el sujeto aporta algo previo a su construcción como objeto, o mejor dicho, algo con lo cual se realiza dicha construcción, la materia del conocimiento: las puras sensaciones?

(ii) La segunda dificultad aparece si analizamos más en detalle el proceso de constitución del objeto. ¿De qué modo las impresiones sensibles que nos rodean dejan de ser datos subjetivos y pasan a constituirse en un mundo objetivo? No hay que perder de vista que este mundo objetivo, el objeto, una vez constituido como tal sigue existiendo únicamente en el sujeto, como algo conocido por el sujeto como contenido mental, igual que la pura sensación. La diferencia es que, frente a la pura sensación, ha adquirido una objetividad que produce la 'falsa apariencia', la 'ilusión', de que existe fuera del sujeto con una constitución en sí, invariable de un sujeto a otro. Estamos en el núcleo de la idea de que el mundo es ilusión. ¿Cómo forma el sujeto esa imagen de un mundo objetivo, a partir de puros datos subjetivos? El objeto consiste en lo que el sujeto pone fuera de sí aplicando el principio de causalidad, al suponer una causa exterior, unificada, de las impresiones subjetivas que él tiene. Con esta errónea aplicación del principio de razón suficiente se produce y alimenta continuamente el contraste entre sujeto y objeto. Veamos ahora la dificultad que queríamos examinar. ¿Significa esto entonces que las sensaciones no vienen del objeto, sino que las tiene el sujeto antes de construirlo como algo exterior? $\mathrm{O}$ en todo caso, ¿qué lugar ocupan las sensaciones entre el sujeto y el objeto?

Siguiendo a Kant, Schopenhauer piensa que el sujeto se limita a aportar las formas a priori con las que se construye la imagen del mundo intuitivo, pero no el contenido, las sensaciones. Kant las denomina 'lo dado', y Schopenhauer critica la insuficiencia de Kant por limitarse a constatar que son dadas sin avanzar en ningún momento la pregunta sobre su origen. En la Crítica a la filosofía kantiana, Schopenhauer resalta que su originalidad está en haber descubierto el origen de 'lo dado' en el conocimiento. No reside en el sujeto, porque es la materia del conocimiento, es aquello que él no pone, que se limita a recibir y a organizar con las formas que él sí pone. Pero en realidad tampoco puede residir en el objeto, porque el objeto en cuanto tal es la imagen mental (Vorstellung) resultante de elaborar eso 'dado' por parte del sujeto. El origen 
de lo dado, de las puras sensaciones, sólo puede estar fuera del fenómeno -que es el ámbito abierto por la relación sujeto-objeto-, en el ser en sí de las cosas: la voluntad.

Con la resolución de estas dificultades, podemos sacar unas cuantas conclusiones acerca del argumento kantiano. Lo habíamos resumido en la idea de que el en acto cognitivo juega un papel preponderante el sujeto frente al objeto, porque es aquél quien organiza la materia del conocimiento. Esta idea era la respuesta a la pregunta-clave para dilucidar el carácter ilusorio del mundo, que habíamos formulado así: ¿qué instancia, en el acto cognitivo, determina predominantemente: el sujeto y sus estructuras, o el objeto y sus estructuras? La respuesta ahora sería de que el objeto no determina nada: ni en cuanto a estructuras, por que en sí mismo no tiene ninguna, sino que le son añadidas; pero tampoco en cuanto al contenido, que no puede provenir de él, sino directamente de la cosa en sí: la voluntad. Es decir, Schopenhauer viene a decir que esa pregunta tradicional de la gnoseología está mal planteada. Pero era la pregunta con la nosotros intentábamos dilucidar las razones que apoyan la tesis inicial de El mundo -el mundo tal como se nos aparece a los sentidos, pero no sólo inmediata sino también mediatamente, es mi representación.

Resumiendo todo lo visto hasta ahora, hemos de concluir que las razones a favor de la ilusoriedad del mundo no pueden ser: ni el idealismo ${ }^{12}$, porque mi subjetividad no interviene completamente en su constitución; ni el argumento del filtro, según el cual el entendimiento interviene en parte preponderante 'filtrando' aquello que viene del objeto, puesto que no hay nada que provenga del objeto que haya que 'filtrar'. El único argumento a favor de la ilusoriedad del mundo debe de salir de comprobar que, según la visión schopenhaueriana, en lo real, en lo en sí, no hay propiamente objeto alguno. La objetividad no es más que un sólido efecto ilusorio dentro de la subjetividad -el paralelo con los sueños es palpable. Pero entonces ¿esta tesis en qué se diferencia del idealismo? En que, a parte de 'lo ideal', Schopenhauer postula también una realidad en sí, no todo se reduce a idea. Todo lo contrario, lo ideal (la idea, el espíritu, el cogito, el yo) no es más que una vana apariencia. Pero entre lo ideal y la realidad en sí no hay ningún 'filtrado', porque ésta última, siendo ser en sí y no para un sujeto, no tiene sin embargo objeto, objetualidad, alguna. En breve, en la cosa en sí no hay objeto alguno, sino que el objeto es una pura invención producida por el sujeto.

Veamos a continuación qué consecuencias importantísimas tiene esta conclusión para entender la concepción schopenhaueriana de la voluntad y de la relación voluntad-representación. Volvamos a la distinción kantiana entre apariencia (fenómeno: Erscheinung) y cosa en sí (Ding an sich). ¿Qué implicaciones tienen las aclaraciones anteriores en la comprensión del concepto de

${ }^{12}$ Sobre el 'idealismo’ schopenhaueriano, cf. A.I. Rábade Obradó, op. cit., pp. 23-41. 
apariencia? Ante todo hay que hacer una distinción semántica importante: el verbo erscheinen, de donde procede Erscheinung, se diferencia del verbo scheinen; el primero significa 'aparecer', mientras que el segundo 'parecer'. En nuestras lenguas romances, tenemos que remitirnos a las raíces latinas. En latín también se distingue parere de ad-parere. Lo importante aquí es que el prefijo ad- o er- da a la raíz parere o scheinen un sentido intransitivo. Así hay que distinguir entre el parecer, donde una cosa parece otra, y el aparecer, donde en principio la cosa se muestra, se hace presente como tal. Una cosa no tiene por qué aparecer 'como otra', sino simplemente aparecer, mostrarse, en contraposición a lo que permanece oculto. Podemos sacar ya una primer conclusión. Al ser en sí del mundo, a la voluntad, no le encaja el significado de parecer, porque no es 'una cosa' con el inconveniente de que se muestra 'como otra', sino que en ningún sentido, ya lo hemos visto, es 'una cosa'. No hay sentido alguno de filtrado. Sí encaja el significado de aparecer: la voluntad se aparece, se muestra, y este aparecerse y mostrarse es el mundo de la representación.

Como siempre, todo este análisis terminológico no sería nada si no fuese acompañado de una comprensión conceptual propiamente dicha. Porque aún el término 'aparecer' implica que se muestra algo que 'antes' estaba 'oculto'. Es decir algo que tiene una forma, unas determinaciones, pero que al principio están ocultas y después, por alguna razón, las manifiesta en mayor o menor medida. Evidentemente este significado también debe ser depurado. No es que la voluntad tenga en sí una esencia que permanece oculta, y a posteriori se manifiesta parcialmente como mundo de la representación, sino que en sí misma no puede tener esencia ni determinación alguna. Por consiguiente, en el ser en sí no hay nada que pueda estar ocultado.

Recojamos ahora la conclusión que había extraído sobre la diferencia entre lo ideal y lo real, y sobre la cosa en sí, para enlazarla con este análisis terminológico y derivar así una primera interpretación del concepto de ilusión. Habíamos visto que el mundo como representación es ilusorio, pero no en el sentido de que posee una forma completamente distinta a la de lo real, al ser en sí, porque el ser en sí la única particularidad que tiene es que no posee forma, esencia alguna. La analogía con los sueños debe aquí cesar completamente, porque los sueños tienen unas formas cuyo sentido reside en su contraposición a la formas reales. Podemos así esbozar una interpretación del concepto de apariencia: la voluntad se muestra, aparece, no porque adquiera otra forma a la que tenía oculta, sino porque en sí misma no tiene forma alguna y adquiere una, y adquiriendo una forma se hace presente, se nos aparece.

Para terminar la cuestión añadiré una aclaración conceptual, no ya terminológica. A mi modo de ver, como en todo gran filósofo, no debemos perder de vista nunca su proceder metodológico. Schopenhauer rechaza proceder mediante la combinación abstracta de los conceptos, como hace el idea- 
lismo, sin que cada nueva conclusión no sea remitida a la experiencia directa. Su posición en este aspecto es radicalmente empirista: los conceptos son abreviaciones de la experiencia, y a ella deben ser remitidos en cada momento si no queremos quedarnos con conocimientos huecos. En el caso que nos ocupa, esto significa que todas estas distinciones y aclaraciones conceptuales que hemos hecho no son meras elucubraciones, sino que pueden y deben se remitidas a alguna experiencia. Para ello debemos buscar la experiencia clave en la que se basa su pensamiento.

Como aparece tematizado extensamente en el Tratado sobre la cuádruple raíz del principio de razón suficiente, Schopenhauer coloca en el centro de su sistema la experiencia del acto del querer, lo que él llama la cuarta clase de objetos para las facultades de la representación. En el acto del querer, yo tengo la experiencia de que mi acto interior de voluntad se manifiesta automáticamente en un acto corporal. Schopenhauer llama a esto el misterio por excelencia de la existencia: en el querer, la interioridad se hace su opuesto, exterioridad. Pienso que la duplicidad conceptual interioridad-exterioridad, basada en esta experiencia, es la oposición básica en que se basa el sistema de Schopenhauer. Esa duplicidad no es analizable más allá, porque remite a una experiencia absolutamente básica, residente en los límites de la representación, que no puede ser reducida a conceptos, porque de ella parten 'todos los conceptos' ${ }^{13}$. Esa duplicidad sólo es 'interpretable' 'más acá', mediante series de conceptos que lo que intentan aclarar lo pierden en contenido ${ }^{14}$.

Con esa duplicidad podemos terminar de entender la relación voluntadrepresentación, y el concepto de apariencia. La voluntad es en sí misma pura interioridad, sin forma alguna, aquella misma que experimento en mí mismo, que adquiere formas, se hace presente, se convierte en exterioridad como actos corporales. Lo verdaderamente real es esa pura interioridad, mientras que el mundo como representación es pura exterioridad, pura apariencia que no se corresponde con nada real porque lo real, la interioridad, no puede tener forma alguna con la que se pueda corresponder.

Concluyamos entonces con la hipótesis interpretativa que me había propuesto poner a prueba. Para Schopenhauer, el mundo como representación es ilusorio en el mismo sentido del concepto kantiano de ilusión: no porque sea distinto a como realmente es, puesto que realmente no posee ninguna determinación, ninguna característica; sino porque 'no tiene objeto': es decir, es objeto, o un conjunto ordenado de objetos, cuando en sí no hay objeto alguno,

${ }^{13}$ R. Malter la califica como 'inmediatamente dada' pero 'absolutamente inconcebible', cf. Der eine Gedanke: Hinführung zur Philosophie Arthur Schopenhauers, Wissenschaftliche Buchgesellschaft, Darmstadt, 1988, p. 32.

${ }^{14}$ El carácter 'hermenéutico' de la metafísica schopenhaueriana ha sido defendido también, argumentando extensamente, por A.I. Rábade Obradó, op. cit., pp. 42-68. 
cuando el en sí es lo más radicalmente distinto a la objetualidad. Pero también es ilusión en sentido kantiano, porque este 'falso efecto' de producir una objetividad cuando en sí misma no existe, no es un efecto que conociéndolo podamos eliminarlo sin más, sino que como las ilusiones ópticas sigue produciéndose ante nuestros ojos. Queda para otro estudio investigar porqué se produce este efecto ilusorio, para lo que hay que recurrir a muchos otros aspectos de la metafísica schopenhaueriana. Así también sería interesantísimo examinar las consecuencias importantes que se derivan de esta hipótesis con respecto a la interpretación que podamos hacer de la voluntad como fundamento y de las relaciones voluntad-representación.

\section{ILUSIÓN, PERSPECTIVA E INTERPRETACIÓN EN NIETZSCHE}

El concepto de ilusión ocupa una posición cardinal en el pensamiento nietzscheano. Así ocurre ya desde El nacimiento de la tragedia, donde el concepto va ligado a la duplicidad schopenhaueriana voluntad-representación. Nietzsche no la toma tal cual, sino que, asimilándola en profundidad, la transforma mediante la dialéctica de las tendencias estéticas de lo apolíneo y lo dionisiaco. Desde el principio, el concepto de ilusión gira en torno a ese concepto fundamental nietzscheano que es el de apariencia (Erscheinung). En relación a éste, el concepto de ilusión (Schein, Illusion) ${ }^{15}$ recalca el aspecto de error (Irrthum), de engaño (Täuschung). Otro concepto de la misma órbita es el de 'ficción' (Fiktion, Erdichtung), que recalca en cambio el aspecto activo de invención, llegando a adquirir el sentido de la ilusión adoptada concienzuda y deliberadamente. Todo este campo conceptual ya lo hallamos desplegado en $E l$ nacimiento de la tragedia, y a partir de la última consideración intempestiva $(R$. Wagner in Bayreuth) comenzará a sufrir profundas transformaciones. A mi modo de ver, ellas obedecen sobre todo a la extensa crítica que a partir de entonces Nietzsche lleva a cabo contra el concepto de 'cosa en sí'. Fuera de la órbita conceptual de lo 'en sî́, el concepto de ilusión adquirirá aspectos y dimensiones completamente nuevos. Aquí estudiaré brevemente el concepto de ilusión tal y como se configura en el pensamiento maduro de Nietzsche.

\footnotetext{
${ }^{15}$ Nietzsche utiliza el término de derivación latina Illusion, que no utiliza Schopenhauer, sobre todo cuando tiene el sentido propio que él le quiere dar. En el período de madurez, el término aparece numerosas veces, aplicado a casos concretos o en general, cf. FW §36, FW $\S 80$, JGB §39, GM III §12, GD «Die 'Verbesserer' der Menschheit» §1, AC §24, KSA IX 434 10[E93], X 254 7[37], X 268 7[77], X 315 7[238], X 507 16[23], X 513 16[43], X 651 24[15], XI 24 25[55], XI 58 25[165], XI 146 25[505], XI 158 26[38], XI 202 26[201], XI 237 26[334], XI 253 26[389], XI 255 26[396], XI 486 34[194], XI 533 35[47], XI 622 39[8], XI 646 40[37], XII 101 2[83], XII 105 2[89], XII 106 2[91], XII 115 2[110], XII 201 5[50], XII 206 5[58], XII 236 6[9], XII 247 7[1], XII 478 10[49], XIII 34 11[72], XIII 226 14[18], XIII 229 14[24], XIII 280 14[103], XIII 415 15[16], XIII 520 17[3].
} 
Para empezar a dilucidar el concepto, podemos observar que su significado básico en la obra nietzscheana es el que hemos anotado desde un principio. Dicho brevemente, una ilusión es un contenido cognitivo que, siendo falso, aparece o se muestra como verdadero, aún después de haber sido reconocida su falsedad. De modo que este contenido cognitivo tiene, por diversas circunstancias, cierta especie de fuerza persuasiva tan intensa que atrapa y embauca nuestro entendimiento. Esto lo podemos entender bien si lo enfocamos desde el sentido que tiene en Nietzsche la tarea crítica. En otro trabajo ${ }^{16}$, dedicado a interpretar la crítica nietzscheana al sujeto del conocimiento, he intentado mostrar in concreto, a lo largo de toda ella, que Nietzsche dirige sus ataques siempre contra 'creencias' o 'convicciones' (Glauben), y nunca contra meros conceptos o proposiciones. La crítica nietzscheana no persigue nunca 'refutar' contenidos cognitivos, es decir, demostrar que son falsos o inconsistentes. Lo que busca, según su célebre expresión en Ecce homo, es 'congelarlos' ${ }^{17}$. La metáfora es sugerente: congelar es neutralizar los intensos efectos persuasivos que ciertos conceptos, proposiciones o teorías han adquirido en la tradición. El problema que le preocupa a Nietzsche es la aceptación pasiva de los 'prejuicios', como una especie de certezas o evidencias que nuestro entendimiento acepta como tales. A Nietzsche no le interesa casi nunca probar, dejar establecida, su falsedad. Lo que le preocupa es conseguir que pierdan ese carácter de 'conocimiento inmediato', y pasen a ser 'conocimientos mediatos' como todos los demás. Por consiguiente, el fenómeno interesante aquí es que hay ciertos contenidos cognitivos que a lo largo de la tradición, por diversas causas, han adquirido una fuerza persuasiva tal que hace que al entendimiento le cueste un enorme esfuerzo y mucho tiempo ponerlos en cuestión para examinarlos de una vez desde su raíz. Esos contenidos cognitivos son entonces, en analogía con el fenómeno óptico, auténticas ilusiones, porque aún reconocida su arbitrariedad o gratuidad, siguen actuando sobre nosotros con su fuerza persuasiva, de manera que fácilmente, por cualquier inadvertencia, volvemos a caer en ellos.

Nos topamos aquí con otro concepto clave nietzscheano, el de 'prejuicio' (Vorurtheil), equivalente al también bastante utilizado de 'presupuesto' (Vorgesetzt) junto al verbo 'presuponer' (vorsetzen). El concepto de ilusión es así una reelaboración de estos conceptos de indudable raigambre moderna. Examinemos con cuidado el término 'prejuicio'. Pre-juicio indica un juicio previo, pero ¿previo a qué? A lo propio de un juicio: previo a su deliberación y emisión conscientes. Es decir, se trata de la idea de que es posible funcionar continuamente con juicios que nunca hemos examinado, sobre los que nunca

${ }^{16}$ M. Parmeggiani, Perspectivismo y subjetividad en Nietzsche, Analecta Malacitana, Málaga, 2002.

${ }^{17}$ Ecce homo, Alianza, Madrid, ${ }^{9} 1985$, pp. 80 y 88. 
hemos hecho el balance de argumentos a favor y en contra, que nunca hemos deducido de manera mínimamente probada. Pero en las teorías acerca de los prejuicios suelen darse dos modalidades que dan razón de su carácter preconsciente:

(i) Los prejuicios como juicios inconscientes: son juicios enclavados de alguna manera en nuestro entendimiento, con los que inevitablemente tiene que funcionar, y que deben su fuerza condicionadora precisamente a que el entendimiento no advierte su presencia, no tiene conciencia de ellos.

(ii) Los prejuicios como certezas o evidencias: si el arma de aquellos es que están ocultos, que son difíciles de desenterrar, la de éstos es la contraria: están demasiado presentes, hasta el punto de que, como ha ocurrido tantas veces a lo largo de la historia y como ha demostrado la ciencia, siendo muy cuestionables y necesitando en todo caso de un examen concienzudo y riguroso, permanecen ahí, ante las conciencias tranquilas, como meras evidencias que 'todo el mundo' puede intuir.

¿Cuál de las dos modalidades hallamos en Nietzsche? En realidad, él utiliza las dos modalidades, propiamente no se decanta por ninguna, por el simple hecho de que su tratamiento de la temática de los prejuicios es de tal naturaleza que las dos aparecen interconectadas. Veamos brevemente cómo ocurre esto. Están las certezas o evidencias que son el blanco directo de la crítica, tanto teoréticas -el cogito, la substancia, la causa final, etc.-, como teóricoprácticas -el bien en sí, la universalidad de la moral, la igualdad, etc. Hemos visto más arriba que la tarea crítica nietzscheana consiste, dicho en pocas palabras, en congelar estos contenidos cognitivos, eliminando de ellos justamente su 'inmediatez'. Creo que a estas alturas del desarrollo es útil introducir entonces el concepto de interpretación, pero sin adentrarnos en las complejidades de la hermenéutica nietzscheana, sino lo suficiente como para aclarar esta cuestión de los prejuicios ${ }^{18}$. En estos términos, la tarea crítica persigue mostrar, hacer ver, patentizar, que esos contenidos cognitivos no son inmediatos, sino que, igual que todos, son interpretaciones. Pero una de las características de las interpretaciones es que siempre son 'mediatas'. ¿Qué significa 'mediato', en contraposición a 'inmediato'? Que los contenidos cognitivos nunca se ofrecen directamente a nuestro entendimiento, sino que para hacerlos presentes, para aclararlos, tenemos que recurrir a otros contenidos que están incluidos implícitamente en los primeros. Por tanto, las interpretaciones son conocimientos 'mediatos' porque siempre contienen presupuestos. En este concepto de 'mediación' se halla contenida la idea de la hermenéutica nietzscheana, que en pocas palabras podemos sintetizar así: todo conocimien-

${ }^{18}$ Sobre la teoría nietzscheana de la interpretación, cf. J. Figl, Interpretation als philosophisches Prinzip. Friedrich Nietzsches universale Theorie der Auslegung im späten Nachla $\beta$, W. de Gruyter, Berlin, 1982. 
to se basa siempre en otros conocimientos previos que da por supuesto, de modo que es imposible un conocimiento completamente apegado a lo real, o que partiese de cero.

La composición del término 'pre-supuesto' nos lo sugiere ya. Supuesto significa 'suposición', es decir, algo que se da por verdadero sin haberlo examinado con cuidado, cuyo sentido es servir como punto de partida para desarrollos ulteriores, de modo que será en ellos donde se revelará la pertinencia o corrección del supuesto. El caso más importante de supuesto es el de la hipótesis. Pero aquí se trata no ya de supuestos, sino de pre-supuestos. Igual que en el caso de los prejuicios, el prefijo indica que son suposiciones que se han hecho de manera completamente previa al acto deliberado y consciente. Descubrimos con ello que los presupuestos corresponden a la primera modalidad de prejuicios, los prejuicios inconscientes. Así podemos establecer la siguiente conexión: los prejuicios como certezas o evidencias ( $2^{\mathrm{a}}$ modalidad) son tales gracias a que incluyen en sí mismos, como sus propios presupuestos, una serie de prejuicios inconscientes. Su función es doble, o más bien se mueve a dos niveles: a un primer nivel, justifican o apoyan la verdad de las evidencias y certezas, y a un nivel más profundo les dan sentido y contenido, de manera que sin ellos ni siquiera sabríamos de qué estamos hablando ${ }^{19}$. Retomando ahora el sentido de la crítica nietzscheana, podemos detallarlo: su tarea es extraer los prejuicios inconscientes que se hallan 'presupuestos' en las supuestas evidencias o certezas, y que les confieren sentido y verosimilitud. Haciendo esto, Nietzsche consigue neutralizar ('congelar') el efecto persuasivo que tienen esas evidencias y certezas sobre el entendimiento, y que pasen a ser otras tantas interpretaciones. Desde luego, la crítica nietzscheana no se agota en hacer eso, pero pienso que, sin entrar en una amplia justificación a través de los textos, constituye lo que podríamos llamar el 'primer nivel' de su desarrollo.

Antes de seguir avanzando, recojamos los resultados obtenidos para ponerlos en conexión con lo que me había propuesto aclarar: el concepto de ilusión. Había caracterizado en un sentido muy general dicho concepto: ilusión es aquel contenido cognitivo que siendo falso, sin embargo se nos aparece como verdadero aún después de haber reconocido su falsedad. Si nos fijamos en el análisis hecho más arriba, con este significado se corresponden las certe-

${ }^{19}$ Es fácil ver aquí el 'círculo hermenéutico' teorizado por Heidegger y la hermenéutica filosófica de Gadamer. No obstante, hay estudiosos (G. Abel, Nietzsche. Die Dynamik der Willen zur Macht und die ewige Wiederkehr, W. de Gruyter, Berlin, 1984) que prefieren diferenciar el planteamiento nietzscheano, donde el concepto cardinal es el de interpretación, del de Gadamer, donde el concepto cardinal es el de comprensión. En esta línea, Günter Abel ha propuesto el 'interpretacionismo', como alternativa a la hermenéutica: Interpretationswelten: Gegenwartsphilosophie jenseits von Essentialismus und Relativismus, Suhrkamp, Frankfurt am Main, 1993. 
zas o evidencias de las que hablábamos. Es decir, aquello que suelen considerarse evidencias o certezas, para Nietzsche son sólo en realidad interpretaciones que han adquirido una fuerza persuasiva tal como para ocultar su naturaleza de conocimiento mediato y derivado. Este efecto persuasivo es en definitiva un efecto ilusorio, son ilusiones, pues igual que en las ilusiones ópticas una imagen errónea se nos aparece como evidente, aún después de haberla reconocido como tal.

Ahora bien, faltaría por aclarar el aspecto de 'falsificación' que contiene el concepto de ilusión, que en cambio no hemos visto hasta ahora en todo el procedimiento con el cual Nietzsche convierte las evidencias y certezas en interpretaciones. Bueno, implícitamente ya se da cierto grado de falsificación en el hecho de que las evidencias y certezas falsifican su propia naturaleza interpretativa: son interpretaciones, pero se muestran o aparecen como lo contrario, como datos inmediatos. Éste es un aspecto bastante elemental que se da también en las ilusiones ópticas: siendo ilusiones, para el entendimiento no avezado son evidencias. Pero igual que en éstas, el aspecto 'falsificatorio' debe residir también a un nivel más profundo. ¿En qué medida las evidencias y certezas no son sólo interpretaciones, sino además interpretaciones 'falsas'?

Podremos resolver esta cuestión si seguimos el hilo conductor que habíamos tomado, la crítica nietzscheana. Lo que he esbozado hasta ahora era sólo un nivel, el más elemental, que cualquier buen lector de Nietzsche sabe que incluye muchas otras cosas. Ahora distinguiré un segundo nivel. Es raro que Nietzsche se conforme con demostrar que las certezas consagradas son en realidad interpretaciones. Lo usual es que dé un paso más para demostrar que además son interpretaciones 'superficiales' (Oberflächlich) de los fenómenos. De esta manera se llega a una neutralización completa de la fuerza ilusoria de esos contenidos cognitivos. Si el primer nivel de crítica ha extraído los presupuestos que revelan esas certezas como interpretaciones, el segundo dirige su ataque a esos presupuestos que sirven de base a las certezas, de modo que demostrando la superficialidad de éstos, su superficialidad repercute, multiplicada, en las certezas mismas.

Antes de continuar aclarando el significado del término 'superficial', conviene esbozar el tercer nivel de crítica, para en lo que sigue tenerlo presente de un vistazo. Una vez que ha demostrado que las certezas son interpretaciones superficiales, su crítica se dirige a ofrecer una explicación de cómo, siendo interpretaciones superficiales, han podido convertirse en certezas y evidencias, es decir, de cómo han adquirido esa fuerza ilusoria de persuasión. Por consiguiente es en esta tercera fase en la que se llega a demostrar que las certezas y evidencias, con las que nos movemos día a día en los ámbitos práctico y teórico, son en realidad 'ilusiones'. Sin querer entrar en más detalles, que alargarían demasiado este trabajo, sólo mencionaré que a mi modo de ver el famoso método genealógico pertenece propiamente a esta tercera fase. Esbozándolo de manera general, su 
objetivo es describir el proceso histórico por el cual meros prejuicios arbitrarios e injustificados se han convertido en certezas e incluso evidencias ${ }^{20}$.

Pasemos entonces al punto más importante para aclarar el significado de ilusión en Nietzsche. Resumamos en una sola frase la definición que he obtenido de 'ilusión': las ilusiones son interpretaciones superficiales que a lo largo de un proceso histórico han adquirido una enorme fuerza persuasiva que las convierte en certezas y evidencias. Pero más precisamente, ¿qué significa 'superficial'? La reacción automática es entender superficial como 'falso'. Nos vemos inducidos a ello además por la analogía con las ilusiones ópticas: tenemos una imagen falsa del objeto que, por diversas causas, se hace pasar por la imagen verdadera. Desde luego, aquí falso y verdadero se entienden en el más estricto sentido ortodoxo, según la concepción de la verdad como correspondencia o adequatio, sea en la tradición aristotélica o en su reelaboración tarskiana. La imagen ofrecida tiene una forma sustancialmente distinta a la forma del objeto real, de modo que no hay correspondencia o adecuación entre ambas, y se dice que es falsa ${ }^{21}$. El problema es que el concepto de falsedad no encaja con la concepción hermenéutica nietzscheana.

La concepción hermenéutica se basa en el cuestionamiento de la antítesis (Gegensatz) verdad/falsedad y del concepto de la verdad como correspondencia, en el que se basa dicha antítesis. Pero si todo contenido cognitivo (percepción, concepto, juicio, etc.) es una interpretación, entonces ¿con respecto a qué criterios podemos hablar de 'falsedad'? Dicho en pocas palabras, falso es lo que no es lo que en realidad es, pero ¿qué ocurre si este 'ser en realidad' es también interpretación? Por ejemplo, Nietzsche habla a menudo de 'falsificación' en el caso de los conceptos referentes a la experiencia interior: voluntad, intención, motivo, libertad, yo, etc. Sus variadísimos análisis psicológicos persiguen mostrar cómo todos esos conceptos son 'falsificaciones' de los estados interiores y los impulsos que configuran la naturaleza humana. Ellos forman el 'sistema vital' del individuo, que es la auténtica realidad de su naturaleza, en contraposición al yo y a la consciencia, que ofrecen de ella una imagen falsificada. Pero ¿qué estatuto de realidad verdadera puede reivindicar ese sistema vital, si todo es interpretación? Dicho en otros términos, ¿cómo puede hablar de falsedad un pensamiento que, metodológicamente, se ha declarado hermenéutico?

Esta contradicción es una más de las famosas paradojas nietzscheanas. Yo esbozaré una vía de solución de ella, pero sólo lo suficiente como para terminar de dilucidar el concepto nietzscheano de ilusión. Aunque todo conoci-

${ }^{20}$ Para casos concretos de este tercer nivel de crítica, remito una vez más a mi trabajo Perspectivismo y subjetividad en Nietzsche, Analecta Malacitana, Málaga, 2002.

${ }^{21}$ Sobre el problema de la verdad y sus paradojas, tenemos un excelente resumen crítico en M. Clark, Nietzsche on truth and philosophy, New York Univ. Press, New York, 1990. 
miento sea interpretación, no debemos concluir de ahí que toda interpretación es igualmente válida. Nietzsche propone a lo largo de su obra otros criterios, distintos a los tradicionales, relacionados con los valores de la vida. Pero no me adentraré en considerar qué otros criterios propone Nietzsche para 'evaluar' las interpretaciones y poder hacer una 'selección' entre ellas.

Mi idea es que esta contradicción se soluciona básicamente teniendo presente el conjunto de términos con el que Nietzsche empareja el de 'falsificación'. Juntos constituyen un campo semántico que podemos englobar bajo un sólo término: simplificación (Vereinfachung). Poniéndolo en relación con las consideraciones anteriores, obtenemos así la tríada 'superficialidad-falsificación-simplificación'. Pienso que hay que tener presente esta cadena en ese mismo orden, de manera que el término siguiente da razón del anterior. Las certezas y evidencias son interpretaciones superficiales porque son falsificaciones de los fenómenos. A su vez, son falsificaciones en tanto que simplifican los fenómenos. Pero ¿por qué el simplificar los fenómenos tiene que abocar en falsificarlos? Yo creo que si observamos atentamente el discurrir del pensamiento nietzscheano observamos que funciona siempre de un modo formalmente igual: a partir de las consideraciones hechas, vemos cómo tal conocimiento es una simplificación, por tanto es una falsificación de los fenómenos. Evidentemente, esta manera de proceder se explica por la visión de la realidad que Nietzsche unas veces da por supuesto, pero que otras explicita claramente: la realidad es cambio y pluralidad radicales, es un incesante 'múltiple fluir'. Entre numerosos textos donde aparece expresada, veamos el siguiente fragmento póstumo de la época de Más allá del bien y del mal: «Interpretación [Auslegung], no explicación. No existen hechos, todo es fluido, inasible, dúctil; lo más duradero siguen siendo nuestras opiniones. Poner adentro sentido [hineinlegen]... en la mayor parte de los casos se trata de una nueva interpretación respecto a una antigua que se ha vuelto incomprensible, que incluso es ya sólo un signo» ${ }^{22}$. Por tanto, simplificar supone siempre falsificar, es decir, reducir el devenir real, negar, obviar su naturaleza esencial. En otras palabras, no puede haber una simplificación 'correcta'. En la concepción substancialista de la metafísica, esto era posible precisamente porque se presuponía una estructura estable en lo real. Pero si lo real es la multiplicidad y el cambio continuo, toda simplificación es 'arbitraria', y por tanto falsa. Denominaré a esta tesis nietzscheana tesis nominalista. No quiero entrar en mayores consideraciones que nos alargarían demasiado, sólo decir que con esta denominación no pretendo emparejarlo, por ejemplo, al empirismo de Hume, del que se diferencia en puntos importantes.

Pero Nietzsche acompaña esta tesis nominalista con otra que me gustaría llamar tesis trascendental (trascendental en sentido kantiano): el conocimien-

${ }^{22}$ KSA XII 100, 2[82]. 
to sólo es posible simplificando ese devenir. Es decir, nuestro entendimiento no puede hacerse cargo de lo real en toda su multiplicidad y mutabilidad, sino que para sacar de él objetos cognitivos (sensaciones, percepciones, conceptos, etc.) tiene forzosamente que simplificarlo, es decir, que reducirlo forzosamente a unos pocos elementos persistentes: «La voluntad de apariencia, de ilusión, de engaño, de devenir y cambio, es más profundamente 'metafísica' que la voluntad de verdad, de realidad, de ser» ${ }^{23}$. Esta tendencia simplificadora del conocimiento se constituye así, para el pensamiento nietzscheano, en un 'a priori', en una especie de condición trascendental del conocimiento, en sentido kantiano; porque es algo que no 'está' en los fenómenos dados, que son pura multiplicidad y puro cambio como hemos visto, en ellos no hay ningún apoyo para esa operación, sino algo que nuestro entendimiento necesita "poner' para poder conocer ${ }^{24}$.

Teniendo presente la tesis nominalista y la trascendental, podemos entonces terminar de clarificar el concepto de 'falsificación'. Yo creo que Nietzsche, como hace casi siempre con los términos de la tradición, juega con el doble sentido de 'falsificación': el sentido tradicional y el sentido transformado por su pensamiento. De manera que pasa continua e inadvertidamente de un sentido a otro. En el sentido tradicional, falsificar es mostrar algo de una forma distinta a como es en realidad. En el nuevo sentido, falsificar es mostrar 'algo', cuando en realidad no hay algo sino si se nos permite la expresión 'muchos algos' que cambian incesantemente. En otras palabras, las interpretaciones falsifican, no porque muestren un objeto como otro objeto, no estando justificada esta conexión; sino porque muestran 'un objeto' donde en realidad no lo hay, donde en realidad lo que hay es un complejo de elementos siempre cambiantes. Veamos un ejemplo: el yo ${ }^{25}$. Esta certeza es una interpretación superficial de nuestra naturaleza en tanto que constituye una 'falsificación' de ella, en el sentido de una simplificación: supone una unidad indivisible y permanente, donde en realidad lo único que hay es una complejo de elementos interrelacionados y que cambian continuamente. Para completar estas aclaraciones, sólo añadiré que, como ya apuntamos, 'superficial' lleva implícita la connotación de 'arbitrario'. Ésta surge en cada momento en que la tarea crítica llega tan lejos como para hacer patente que las interpretaciones no son sencillamente simplificaciones, sino simplificaciones 'excesivas' del devenir, y por tanto inevitablemente arbitrarias.

${ }^{23}$ KSA XIII $22614[18]$.

${ }^{24}$ En este 'apriorismo' de Nietzsche insiste mucho N. Davey ( «Nietzsche and Hume on Self and identity», The Journal of the British Society for Phenomenology, 18, 1987, 14-29), pues en él encuentra la diferencia radical que lo distancia de Hume y una contradicción cardinal de su gnoseología.

${ }^{25}$ Cf. mi libro Nietzsche: Crítica y proyecto desde el nihilismo, Ágora, Málaga, 2002, cap. VII, pp. 131-160. 
Creo que con esta caracterización de la falsificación podemos ya resolver la contradicción que se nos había presentado. Para ello basta con tener presente lo que se deriva del concepto de falsificación como simplificación: para evaluar un contenido cognitivo como falso, Nietzsche, a pesar de lo que pueda parecer, no recurre a contraponerlo a otro verdadero. Es decir, no es como si dijera: el objeto considerado dicen que es así, pero en realidad es de otra manera. Sino como si dijese: dicen que el objeto es así, pero en realidad lo que ocurre es que 'no hay objeto', sino una multiplicidad cambiante que habrá que considerar parte por parte, y a través de sus largos procesos temporales.

Podría replicarse que esa imagen del devenir es ya una imagen o interpretación verdadera, en el sentido de correcta, de lo real, de modo que Nietzsche vuelve a caer en la contradicción: contraponer un contenido cognitivo verdadero cuando previamente su teoría hermenéutica ha negado la existencia de toda verdad. A mi modo de ver esta réplica mezcla sofísticamente los distintos niveles lógicos ${ }^{26}$. Básicamente por dos razones:

(i) Hay que distinguir entre un nivel más bajo, 'objetual', en el que se describe concretamente el objeto. Por ejemplo, en la antropología cartesiana, el hombre posee un yo que está constituido por la autoconciencia. Y otro nivel superior, 'metaobjetual', en el que no se da ninguna descripción concreta, porque se habla, no del objeto, sino de las descripciones que se intenta dar de él: afirmando que ninguna descripción es válida porque no hay objeto alguno que describir. En el ejemplo propuesto, la antropología metafísica se mueve en el nivel objetual, mientras Nietzsche desarrolla su crítica desde un nivel superior 'metaobjetual'. Por tanto, las afirmaciones de Nietzsche no son 'verdaderas' en el mismo sentido en que lo son las de la antropología metafísica. Al pertenecer a un nivel lógico superior, las aserciones de la crítica nietzscheana no pertenecen al grupo de 'descripciones más verdaderas del objeto', no versan directamente sobre el objeto, sino sobre las relaciones que intermedian entre las descripciones y el objeto.

(ii) Esta distinción significa que hay que entender de modo correcto las tesis nietzscheanas. Veámoslo con un caso concreto para no perdernos en generalidades. Cuando Nietzsche contrapone a la interpretación metafísica de la naturaleza humana bajo el concepto de yo, su propia interpretación como 'sistema vital de impulsos', no está contraponiendo a una descripción concreta otra descripción concreta más verdadera, sino sencillamente a una descripción concreta, una serie de criterios metodológicos de investigación más fructíferos para acercarse a lo real. Deducir de ahí que a la vez nos propone una

${ }^{26}$ Este distinción de niveles lógicos va dirigida a invalidar la acusación de inconsistencia que se ha hecho a su teoría de la verdad, pero reconozco que no es una réplica sólida a la objeción que hace Rorty, de una 'autocontradicción performativa', cf. Contingency, irony, and solidarity, Cambridge University Press, Cambridge, 1989. 
'descripción verdadera' es malentender el proceder de su pensamiento. Es decir, bajo su crítica no hay que sobrentender la afirmación dogmática «la naturaleza humana no es como dicen ellos sino como digo yo», sino el criterio metodológico: «allí donde la investigación se haya detenido en una 'substancialización' (la unidad del yo), no te detengas, sino sigue intentando sacar a la luz, pacientemente, la pluralidad y el cambio que hay detrás». Pienso que sólo bajo este punto de vista hay que entender las descripciones concretas que desde luego ofrece Nietzsche incesantemente: como propuestas para sacar a la luz más y mejor el devenir. Pero en cuanto descripciones concretas también ellas, aunque en menor grado, simplifican el devenir, y por tanto caen bajo el efecto de ilusión ${ }^{27}$.

En definitiva, hemos llegado a lo que me había propuesto demostrar: que detrás del concepto de ilusión nietzscheano, igual que detrás del shopenhaueriano, está el concepto kantiano de ilusión trascendental. Lo que quiero decir es que la mejor manera de entender el primero, para evitar las paradojas y los malentendidos, es hacerlo según el esquema del segundo: Nietzsche califica las certezas de ilusiones en el mismo sentido en que lo entiende Kant: no porque muestren lo real como no es, sino porque suponen un objeto donde en realidad no hay ninguno, tenga la forma que tenga. Es decir, igual que las ilusiones trascendentales, las ilusiones nietzscheanas 'no poseen objeto', más aún, 'no pueden poseer objeto', ya que en la realidad no hay forma sólida alguna, ninguna 'cosa', ninguna 'substancia'. Igual que en Kant, no es una cuestión empírica, sometida a la contingencia de la investigación. La ilusión está, no en producir un objeto distinto al real, sino en el simple hecho ya de producir un objeto, porque frente al fluir incesante del devenir todo objeto es ficticio. Además, igual que en Kant, la ilusión de que hay objeto, donde no lo puede haber, se sigue produciendo siempre, aún después de ser reconocida; puesto que nuestro entendimiento, nuestro pensamiento, no puede afrontar el caos del devenir tal cual, en su cambio y multiplicidad radicales, sino que tiene necesariamente que crear objetos en el devenir, tiene que solidificarlo.

\footnotetext{
${ }^{27}$ Se concluirá entonces que volvemos al principio, que qué sentido tiene dar tantas vueltas para terminar siempre en ilusiones. Y si embargo, observando atentamente, vemos que sí se ha hecho un gran avance. Porque, como hemos dicho, simplifican pero en menor grado. Es decir, lo importante son los grados. Los términos absolutos son ficticios, sólo hay grados de verdad y falsedad. En términos absolutos, todo es falso o todo es verdadero. En la época de Más allá del bien y del mal hallamos explícitamente confirmada esa conclusión: «Ya no encontramos que haya oposición entre 'realidad' y 'apariencia', hablaríamos en todo caso de grados del ser, o mejor aún, de grados de ilusión [Graden des Scheins]» KSA XI 637 40[20].
} 